\title{
Budesonide use and misuse in sports: elimination profiles of budesonide and metabolites after intranasal, high-dose inhaled and oral administrations.
}

Sergi Coll ${ }^{1,2}$ (ORCID: 0000-0003-0729-4470), Nuria Monfort ${ }^{1}$, Xavier Matabosch ${ }^{1}$, Katerina Papakonstatinou ${ }^{1}$, Clara Pérez-Mañá ${ }^{3,4}$, Julian A Mateus ${ }^{5}$, Rosa Ventura $^{1,2}$ (ORCID: 0000-0002-1413-8890)

(1) IMIM (Hospital del Mar Medical Research Institute), Doping Control Research Group, Catalonian Antidoping Laboratory, Barcelona, Spain

(2) Department of Experimental and Health Sciences, Universitat Pompeu Fabra, Barcelona, Spain

(3) Department of Clinical Pharmacology, Hospital Universitari Germans Trias i Pujol (HUGTP-IGTP), Badalona, Spain

(4) Department of Pharmacology, Therapeutics and Toxicology, Autonomous University of Barcelona, Cerdanyola del Vallès, Spain

(5) IMIM (Hospital del Mar Medical Research Institute), Human Pharmacology and Clinical Neurosciences Research Group, Barcelona, Spain

Corresponding author: Rosa Ventura Alemany

Doctor Aiguader 88, 08003 Barcelona, Spain

E-mail: rventura@imim.es

Phone: +0034-933160471 Fax: +0034-933160499 
Abstract

Budesonide (BUD) is a glucocorticoid (GC) widely used in therapeutics. In sports, the World Anti-doping Agency (WADA) controls the use of GCs, and WADA-accredited laboratories use a reporting level of $30 \mathrm{ng} / \mathrm{mL}$ for $6 \beta$-hydroxy-budesonide (6ßOHBUD) to detect the systemic administration of BUD. In the present work, we examined the urinary excretion profile of $6 \beta \mathrm{OHBUD}, \mathrm{BUD}$, and 16 $\alpha$-hydroxy-prednisolone (16 $\alpha$ OHPRED) after intranasal (INT), inhaled (INH) (at high doses) and oral administrations in male and female volunteers.

BUD was administered to healthy volunteers using INT route $(256 \mu \mathrm{g} / \mathrm{day}$ for three days, $n=4$ males and 4 females $)$, INH route $(800 \mu \mathrm{g} /$ day for three days, $n=4$ males and 4 females and $1600 \mu \mathrm{g} /$ day for three days, $\mathrm{n}=4$ males) or oral route $(3 \mathrm{mg}, \mathrm{n}=8$ females). Urine samples were collected before and after administration at different time periods, and were analysed by liquid chromatography-tandem mass spectrometry.

$6 \beta \mathrm{OHBUD}$ and BUD concentrations were very low after INT treatment (0.0-7.1 and 0.0-8.1 ng/mL, respectively), whereas they were higher after INH treatments $(0.0-35.4$ and $0.0-48.3 \mathrm{ng} / \mathrm{mL}$, respectively). For $16 \alpha$ OHPRED, elevated concentrations were detected after INT and INH treatments $(2.6-66.4$ and $3.4-426.5 \mathrm{ng} / \mathrm{mL}$, respectively). Concentrations obtained following oral administration were higher than after therapeutic administrations $(2.8-80.6,1.5-36.1$ and $10.4-532.2 \mathrm{ng} / \mathrm{mL}$ for $6 \beta O H B U D$, BUD and 16 $\alpha$ OHPRED, respectively). After all administrations, concentrations were higher in males than in females.

Results demonstrated that $6 \beta$ OHBUD is the best discriminatory marker and a reporting level of $40 \mathrm{ng} / \mathrm{mL}$ was found to be the best criterion to distinguish allowed from forbidden administrations of BUD. 


\section{Introduction}

Budesonide (BUD) is a glucocorticoid (GC) commonly used in therapeutics by different administration routes such as inhalation (INH), in the treatment of asthma (1), intranasal (INT), in the treatment of rhinitis (1), and oral administrations, for the inflammatory bowel disease (2).

GCs are prohibited in sports because of evidences of positive effects on exercise performance and health risks associated to their use due to the inhibition of the hypothalamic-pituitary-adrenal axis (3-5). GCs are prohibited in competitions when administered by systemic routes (oral, intramuscular, intravenous or rectal routes), and they are allowed for therapeutic purposes using other administration routes considered of local action (INT and INH adminsitrations, among others).

World Anti-Doping Agency (WADA)-accredited laboratories detect the systemic administration of BUD by targeting its metabolite, 6 $\beta$-hydroxy-budesonide (6ßOHBUD), with a reporting level of $30 \mathrm{ng} / \mathrm{mL}$ (6). This criterion was established based on studies performed in our group $(7,8)$. First, the urinary metabolic profile of

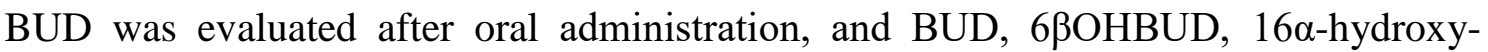

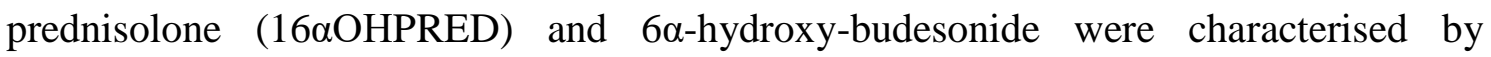
comparison with reference materials (7). Moreover, twelve BUD metabolites were identified and their tentative structures were proposed based on mass spectrometric data (7). Second, the urinary excretion profiles of BUD metabolites were evaluated after oral and INH adminsitrations to identify the best criterion to discriminate between authorized and forbidden administrations of BUD (8). In that study, it was demonstrated that using a reporting level of $30 \mathrm{ng} / \mathrm{mL}$ of $16 \alpha \mathrm{OHPRED}$, the main metabolite of BUD, false adverse analytical findings (AAFs) are detected after allowed INH administration. $6 \beta$ OHBUD was found to be the best discriminatory marker (8), and was implemented in 
WADA regulations (6). The adoption of the new marker drastically reduced the number of AAFs reported by WADA accredited laboratories $(9,10)$.

In our previous work (8), the clinical studies performed consisted of oral and standard INH doses, and only male subjects were recruited for both treatments. Since INT and high-dose INH treatments of BUD are also widely prescribed (1), it is of utmost interest to study the urinary excretion profiles of BUD metabolites after these administration routes. Moreover, it is important to the study the metabolism of BUD in female subjects in order to evaluate potential gender differences.

The aim of the present work was to provide more data about the metabolism of BUD to improve the detection of its systemic use in sports. For that purpose, the urinary excretion profile of BUD and the most important metabolites, $6 \beta$ OHBUD and 16 $\alpha$ OHPRED (Figure 1S, supplementary information), were studied after INT, highdose INH and oral administrations to healthy male and female volunteers. 


\section{Experimental}

\subsection{Clinical studies}

Two clinical studies that involved different administrations of BUD to healthy Caucasian subjects were performed (Table 1). (protocol codes IMIMFTCL/DACORSIN/2 and IMIMFTCL/DACORSIN/3). Ethical approval of the clinical studies was granted by the local Research Ethical Committee (Comitè Etic d'Investigació Clínica CEIC-Parc de Salut Mar, Barcelona, Spain) and the Spanish Medicines Agency (EudraCT protocol numbers 2014-002862-54 and 2017-004839-37, respectively).

A total of sixteen healthy volunteers, twelve females and four males, were recruited (Table 2). All participants gave their written informed consent prior inclusion and underwent a general physical examination, routine laboratory test, urinalysis, and a 12lead electrocardiogram with results within normal values. All volunteers declared not to consume drugs that could alter GCs metabolism such as steroids, prohormones, contraceptives or diuretics.

The first clinical study was a cross-over clinical trial where four males and four females received INT and high-dose INH treatments (Table 1). Wash-out periods of 10 days were carried out between treatments. First, volunteers underwent an INT therapy that involved the administration of a daily dose of $256 \mu \mathrm{g}$ (two sprays of $64 \mu \mathrm{g}$ in each nostril) (Rhinocort ${ }^{\circledR}$, AstraZeneca, Spain) for three consecutive days. The daily dose was administered as a single dose on day 1 and as half dose every $12 \mathrm{~h}$ on days 2 and 3 . Second, subjects received an INH treatment that consisted of a daily dose of $800 \mu \mathrm{g}$ (Pulmicort Turbuhaler®, AstraZeneca, Spain) for three consecutive days. Finally, male volunteers underwent an INH treatment with a daily dose of $1600 \mu \mathrm{g}$ (Pulmicort Turbuhaler®, AstraZeneca, Spain). In both INH therapies, doses were administered as 
half dose every $12 \mathrm{~h}$ for three consecutive days. Urine collection periods were the same for all three treatments: pre-administration and after the first administration from $0-6 \mathrm{~h}$, 6-12 $\mathrm{h}$ and 12-24 $\mathrm{h}$ on days 1,2 and 3, and from $0-12 \mathrm{~h}$ and $12-24 \mathrm{~h}$ on day 4.

The second clinical study consisted of a single-arm trial, where a single oral dose of 3 mg (sustained release capsules, Entocord; AstraZeneca, London, United Kingdom) was administered to eight female volunteers (Table 1). The oral treatment administered was the same as the one used in our previous study with male volunteers (8). Urine samples were collected before administration and after administration from 0-4 h, 4-8 h, 8-12 $\mathrm{h}$ and $12-24 \mathrm{~h}$ on day 1 , from $0-12 \mathrm{~h}$ and $12-24 \mathrm{~h}$ on day 2 , and from $0-24 \mathrm{~h}$ on days 3 and 4.

Urine samples were collected in sterile containers. Preliminary measurements, including specific gravity (SG) and $\mathrm{pH}$, were performed. SG was determined using an Atago UG$\alpha$ Refractometer (Atago, Tokyo, Japan), and pH was measured by a Crison GLP $21 \mathrm{pH}$ meter (Crison, Alella, Spain). Urine samples were stored at $-20^{\circ} \mathrm{C}$ until further analysis.

\subsection{Chemical and Reagents}

Budesonide (BUD) and ammonium formate (analytical grade) were obtained from Sigma (St Louis, MO, USA). 16 $\alpha$-hydroxy-prednisolone (16 $\alpha$ OHPRED), 6 $\beta$-hydroxy-

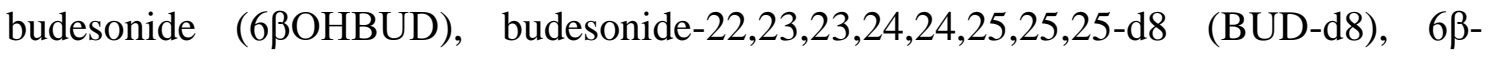
hydroxy-budesonide-22,23,23,24,24,25,25,25-d8 (6ßOHBUD-d8), and 16 $\alpha$-hydroxyprednisolone-11,12,12,21,21-d5 (16 $\alpha$ OHPRED-d5) were purchased from Toronto Research Chemicals (Toronto, Canada). The $\beta$-glucuronidase preparation (type Escherichia coli K12) was purchased from Roche Diagnostics, GmbH (Manheim, Germany). Analytical grade disodium hydrogen phosphate, sodium hydrogen 
phosphate, potassium carbonate, ethyl acetate, acetonitrile and methanol (liquid chromatography gradient grade), and formic acid (liquid chromatrography-mass spectrometry grade) were obtained from Merck (Darmstadt, Germany). Milli-Q water was obtained using a Milli-Q purification system (Millipore Ibérica, Barcelona, Spain).

\subsection{Sample Treatment}

An extraction protocol previously described was used (8). Internal standards (ISTDs) (20 ng of BUD-d8, $40 \mathrm{ng}$ of $16 \alpha$ OHPRED-d5, and $40 \mathrm{ng}$ of $6 \beta$ OHBUD-d8) were added to $2 \mathrm{~mL}$ of urine, followed by the addition of $500 \mu \mathrm{L} 1 \mathrm{M}$ phosphate buffer, $\mathrm{pH}$ 7. Then, enzymatic hydrolysis was performed adding $30 \mu \mathrm{L}$ of $\beta$-glucuronidase enzyme from Escherichia coli, and the mixture was incubated for $1 \mathrm{~h}$ in at $55^{\circ} \mathrm{C}$ in a water bath. The buffered solution was alkalinized with $150 \mu \mathrm{L}$ of $25 \%$ potassium carbonate solution to $\mathrm{pH} 8-9$, and the steroids were extracted with $6 \mathrm{~mL}$ of ethyl acetate. After centrifugation (5 minutes at $1400 \mathrm{~g}$ ), the organic layer was transferred into a new tube and evaporated to dryness under a nitrogen stream in a water bath at $40^{\circ} \mathrm{C}$. The residue was reconstituted into $100 \mu \mathrm{L}$ of a mixture of water to acetonitrile $(1: 1, \mathrm{v} / \mathrm{v})$.

\subsection{LC-MS/MS analysis}

Liquid chromatography-tandem mass spectrometry analyses were carried out using a triple quadrupole (Xevo TQ-S micro) mass spectrometer provided with an orthogonal Z-spray-electrospray interface (Waters Associates, Mildford, MA) coupled to an Acquity ultra-performance liquid chromatographic system (Waters Associates) for the chromatographic separation. Nitrogen and argon (both with purity $\geq 99.999 \%$ ) were used as desolvation and collision gases, respectively. The desolvation and cone gas flows were set to approximately $1200 \mathrm{~L} / \mathrm{h}$ and $50 \mathrm{~L} / \mathrm{h}$, respectively. The nitrogen desolvation 
temperature was set to $450{ }^{\circ} \mathrm{C}$ and the source temperature to $120^{\circ} \mathrm{C}$. A capillary voltage of $3.5 \mathrm{kV}$ was used in positive ionization mode.

The liquid chromatographic separation was performed using an Acquity BEH C18 column (100 x $2.1 \mathrm{~mm}$ internal diameter, $1.7 \mu \mathrm{m}$ particle size) (Waters Associates) using a column temperature of $45^{\circ} \mathrm{C}$. Ammonium formate $1 \mathrm{mmol} / \mathrm{L} \mathrm{pH} 3$ (formic acid) (solvent A) and acetonitrile with formic acid (0.01\%) (solvent B) were selected as mobile phase solvents. The percentage of organic solvent was linearly changed as follows: 0 min, $10 \% \mathrm{~B} ; 0.5 \mathrm{~min}, 10 \% \mathrm{~B} ; 7 \mathrm{~min}, 50 \% \mathrm{~B} ; 8.7 \mathrm{~min}, 90 \% \mathrm{~B} ; 8.8 \mathrm{~min}, 10 \%$ $\mathrm{B}$; and $10 \mathrm{~min}, 10 \% \mathrm{~B}$ at a flow rate of $0.6 \mathrm{~mL} / \mathrm{min}$. The injection volume was $10 \mu \mathrm{L}$. Mass spectrometric analysis was performed in selected reaction monitoring mode by monitoring at least two transitions for each metabolite in the conditions described in Table 3.

\subsection{Statistical Analysis}

Statistical analysis was performed using computer software SPSS 12.0 (SPSS Inc., Chicago, IL, USA). Mann-Whitney U test was used to compare concentrations of BUD metabolites when two different INH doses were administered as well as when BUD was INT administered as a single dose or in two divided doses. The level of significance was established at $\mathrm{p}<0.05$.

In order to correct urinary concentrations by urine dilution, the concentrations were adjusted to a reference value of 1.020 following the Levine-Fahy equation $(11,12)$ :

$$
C_{\mathrm{SG}}\left(\frac{n g}{m L}\right)=C\left(\frac{n g}{m L}\right) \times\left(\frac{1.020-1}{S G_{\text {sample }}-1}\right)
$$

Using the formula, SG-correction will lead to an increase of concentrations when samples present SG values lower than 1.020. 
3 Results

6ßOHBUD, BUD, and 16 $\alpha$ OHPRED were measured in all samples collected after INT, INH and oral administrations. The sample preparation procedure applied allows for the detection of the sum of free and glucuronoconjugated metabolites. 16aOHPRED was the main metabolite after all administrations studied, as reported previously (8).

\subsection{Intranasal and inhaled administrations}

Concentrations of $6 \beta$ OHBUD, BUD, and 16 $\alpha$ OHPRED obtained after INT and highdose INH administrations are presented in Figure 1 and are also described in Table 1S of supplementary information. For INT and INH treatments, metabolites were detected on days 1 to 3 , when subjects were under treatment, and were almost undetectable on day 4, when there was no administration. After INT treatment, 6 6 OHBUD and BUD concentrations were very low (0.0-7.1 and 0.0-8.1 ng/mL, respectively). Concentrations obtained after INT administration did not show statistical differences when BUD was administered as a single daily dose or in two divided doses. After both high-dose INH

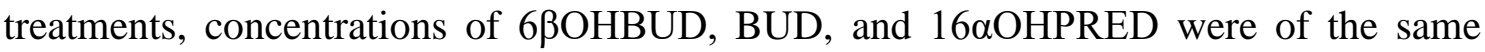
order. The majority of the samples collected after INH treatments presented $6 \beta$ OHBUD and BUD concentrations lower than $10 \mathrm{ng} / \mathrm{mL}$ (Figure 1), however some samples gave elevated concentrations (ranges $0.0-35.4$ and $0.0-48.3 \mathrm{ng} / \mathrm{mL}$ for $6 \beta$ OHBUD and BUD, respectively) (Figure 1 and Table 1S). For 16 $\alpha$ OHPRED, high concentrations were obtained after both INT and INH treatments (2.6-66.4 and 3.4-426.5 ng/mL, respectively).

After both INT and INH treatments, higher concentrations were found in males than in females, especially for 6ßOHBUD and BUD (Figure 1 and Table 1S). 
In order to assess the variability due to urine dilution, urinary concentrations were SGcorrected (Figure 2S, supplementary information). Post-administration urines presented SG values in the range of 1.003-1.030 and 1.004-1.023, for males and females, respectively. While SG-corrected concentrations of males were of the same order than concentrations obtained without correction, SG-normalization led to a much higher concentrations for females. Therefore, the gender differences previously observed were reduced when concentrations were normalized by SG.

The impact of SG-correction on the reduction of gender differences was more noticeable in the INH than INT samples, probably because $6 \beta$ OHBUD and BUD were not detected in some female samples collected after INT administration (LOQ 0.5 and $0.03 \mathrm{ng} / \mathrm{mL}$ for $6 \beta O H B U D$ and BUD, respectively (8)). Therefore, the effect of SGadjustment could not be evaluated on those INT samples.

The urinary excretion profiles of $6 \beta$ OHBUD, BUD, and 16 $\alpha$ OHPRED after INT and high-dose INH administrations are presented in Figure 2. The profiles of the excretion rates obtained after INT and INH administration routes were very similar, however excretion rates were higher after INH administration. On days 1 to 3 , excretion rates showed a consequent increase after the administration of each dose, and a rapid recovery in the post-administration hours. After the last dose on day 3, excretion rates linearly decreased, showing a complete elimination on day 4. Due to a high interindividual variability, clear gender differences were not observed in the excretion rates of BUD metabolites (Figure 2).

\subsection{Oral administration}

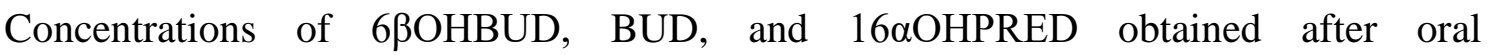
administraton to females are presented in Figure 3 and are also described in Table $1 \mathrm{~S}$ of 
supplementary information. For comparison purposes, data from our previous study (8), where the same oral dose was administrated to males, are included.

Elevated concentrations were found only on day 1 (2.8-80.6, 1.5-36.1 and 10.4-532.2 $\mathrm{ng} / \mathrm{mL}$ for $6 \beta$ OHBUD, BUD and $16 \alpha$ OHPRED, respectively). In this time period, concentrations were higher in males than in females (Figure 3 and Table 1S).

Concentrations were corrected by SG in order to evaluate the variability due to urine dilution. SG values obtained in samples collected during the first day after oral administration, when BUD metabolites were mainly detected, were in ranges of 1.0021.032 and 1.004-1.025 for males and females, respectively. After SG-normalization, gender differences were reduced because female concentrations considerably increased and concentrations of males were of the same order as the non-corrected ones (Figure 3S, supplementary information).

The urinary excretion profiles of $6 \beta \mathrm{OHBUD}$, BUD, and $16 \alpha \mathrm{OHPRED}$ after oral administration are depicted in Figure 2. Excretion rates increased and reached the maximum values in samples collected $0-8 \mathrm{~h}$ after administration, and linearly decreased till being completely eliminated in the third day. After oral administration, excretion rates of BUD metabolites were similar for females and males (Figure 2). The percentages of the dose of the different metabolites detected during the first $72 \mathrm{~h}$ obtained in females were similar to those obtained for males (8), representing $1.4 \pm 0.9 \%$, $0.7 \pm 0.3 \%$, and $11.4 \pm 2.1 \%$ of the dose for $6 \beta$ OHBUD, BUD, and $16 \alpha \mathrm{OHPRED}$, respectively. 
3.3. Discrimination between allowed and forbidden administrations

The suitability of the current WADA criterion to detect BUD misuse was evaluated. For that purpose, the percentage of AAFs detected using the reporting level of $30 \mathrm{ng} / \mathrm{mL}$ for 6ßOHBUD were calculated after different BUD administrations (Table 4A). Data obtained in a previous study that involved standard-dose INH treatment (400 $\mu \mathrm{g} /$ day/3days) and oral administration to male volunteers were also included in the evaluation. As can be seen in Table 4A, false AAFs are detected after high-dose INH administrations because two male samples presented 6ßOHBUD concentrations higher than $30 \mathrm{ng} / \mathrm{mL}$ : One sample out of the 36 samples (3\%) collected after INH $800 \mu \mathrm{g} / \mathrm{day}$ treatment and one sample out of the 36 samples (3\%) collected after INH $1600 \mu \mathrm{g} / \mathrm{day}$ treatment with $6 \beta$ OHBUD concentrations of 35.4 and $31.0 \mathrm{ng} / \mathrm{mL}$, respectively. BUD concentrations higher than $30 \mathrm{ng} / \mathrm{mL}$ were also found in two samples collected after high-dose INH treatments (Table 4A). One sample had concentrations of both $6 \beta O H B U D$ and BUD higher than $30 \mathrm{ng} / \mathrm{mL}$. Therefore, a reporting criterion of 30 $\mathrm{ng} / \mathrm{mL}$ for either $6 \beta \mathrm{OHBUD}$ or BUD would lead to false AAFs after high INH doses (Table 4A).

Reporting levels that ensured no false AAFs after INH were proposed for each marker (Table 4A). The proposed reporting levels were 40,50 and $450 \mathrm{ng} / \mathrm{mL}$ for $6 \beta O H B U D$, BUD, and $16 \alpha \mathrm{OHPRED}$, respectively, and they were chosen taking into account the maximum concentrations obtained after allowed INT and INH administrations (35, 48 and $427 \mathrm{ng} / \mathrm{mL}$, respectively). Results showed that the best criterion to detect BUD misuse is a reporting level of $40 \mathrm{ng} / \mathrm{mL}$ for $6 \beta \mathrm{OHBUD}$ (Table $4 \mathrm{~A}$ ), with no false AAF after INT and INH treatments and the highest number of AAFs after oral administration. Due to the fact that concentrations obtained after oral adminsitration were lower for 
females than for males, the percentage of AAFs detected for females is lower (Table 4A).

In order to assess the possibility to establish a reporting level based on SG-normalized concentrations, the percentage of AAFs obtained using reporting levels based on SGcorrected concentrations were calculated (Table 4B). Since concentrations increased after SG-correction, the reporting levels to avoid false AAFs after INT and INH adminsitrations had to be higher. The maximum SG-corrected concentrations obtained after INT and INH doses were 45, 44, and $426 \mathrm{ng} / \mathrm{mL}$ for $6 \beta O H B U D, B U D$, and 16 $\alpha$ OHPRED, respectively. Therefore, the reporting levels proposed were 50, 50 and $450 \mathrm{ng} / \mathrm{mL}$ for $6 \beta$ OHBUD, BUD, and $16 \alpha$ OHPRED, respectively. Thus, the current reporting criterion of $30 \mathrm{ng} / \mathrm{mL}$ for $6 \beta \mathrm{OHBUD}$ produced a higher number of false AAF when SG-corrected concentrations are monitored (Table 4B). Five samples presented SG-corrected concentrations of $6 \beta O H B U D$ higher than $30 \mathrm{ng} / \mathrm{mL}$ after high-dose INH treatments (3 samples (8\%) after INH $800 \mu \mathrm{g} /$ day treatment and 2 samples $(6 \%)$ after INH $1600 \mu \mathrm{g} /$ day treatment). Results obtained showed that the detection of AAFs after oral ingestion is very similar between males and females (Table 4B).

Ratios between metabolites were also studied to look for differences between routes of administration, but they did not improve the detection of systemic BUD. 


\section{Discussion}

In this work, the urinary excretion profiles of $6 \beta$ OHBUD, BUD and 16 $\alpha$ OHPRED following INT, high-dose INH and oral administrations are described. In all treatments, recommended therapeutic dosages were used $(1,2)$. INT and INH doses administered were the maximum dosages recommended in therapeutics. In general, INT and INH treatments prescribed in therapeutics are longer than the ones performed in the present study (1). However, the administration of BUD by either INT or INH routes for three consecutive days was enough to evaluate a potential drug accumulation, and the impact on anti-doping controls. On the other hand, the oral treatment was a short and low-dose therapy (2), which allowed the evaluation of the minimum number of AAFs that could be detected following oral administration.

As expected, excretion rates obtained after oral administration were higher than after INT and INH treatments (Figure 2). Excretion rates obtained after INH administrations were greater than after INT treatment probably because INH doses were much higher. Since no accumulation was seen after multiple INT or INH administrations for three consecutive days, the results obtained in the present study could be extrapolated to longer INT and INH treatments.

Results obtained in the present work confirms that $6 \beta$ OHBUD is the best marker to discriminate allowed from forbidden administrations of BUD (Table 4A). Interestingly, 6ßOHBUD and BUD concentrations obtained after INT and INH administrations were very similar (Figure 2), but 6ßOHBUD was excreted in higher amounts than BUD after oral administration (Figure 3). For this reason, 6 $60 \mathrm{OHBUD}$ is a better discriminatory marker than BUD. However, using a reporting level of $30 \mathrm{ng} / \mathrm{mL}$ for $6 \beta$ OHBUD false AAFs may be reported after high-dose INH administrations. A reporting level of 40 
$\mathrm{ng} / \mathrm{mL}$ for $6 \beta$ OHBUD was found to be the best discriminatory criterion (Table 4A). Although increasing the reporting level would suppose a lower detection of AAFs after oral use, it is of paramount importance to avoid the possibility of reporting false AAFs after INT and INH treatments due to the wide use of BUD using these administration routes for therapeutic purposes. Moreover, BUD has reduced systemic effects (1), and therefore, it is not reasonable to expect that athletes use oral BUD for doping purposes. Nonetheless, it has to be taken into account that the oral therapy administered was a short and low-dose treatment, and therefore, the likelihood of considering a sample as AAF when it is collected during or after a high-dose treatment should be higher. On the other hand, it is re-confirmed that the use of a reporting level of $30 \mathrm{ng} / \mathrm{mL}$ for $16 \alpha \mathrm{OHPRED}$ is not adequate, since a large number of AAFs were detected after allowed INT and INH administrations.

The possibility to establish a reporting level based on SG-corrected concentrations was evaluated, however the discrimination between allowed and forbidden adminsitrations of BUD worsened. On the one hand, a higher number of false AAFs were detected after INH using the current reporting level of $30 \mathrm{ng} / \mathrm{mL}$ for $6 \beta O H B U D$ (Table 4B). On the other hand, a lower number of male samples was considered as AAF after oral administration in comparison to the results obtained using the proposed reporting level of $40 \mathrm{ng} / \mathrm{mL}$ for non-corrected concentrations of $6 \beta$ OHBUD (Table 4B). Therefore, the recommendation is to use a reporting level based on non-corrected concentrations, even though it may present difficulties to consider as AAF a diluted urine collected after oral administration. 
To our knowledge, it is the first time that the urinary metabolism of BUD is studied in women, and it was found that concentrations of BUD metabolites were lower in females than in males. Interestingly, SG values of the post-administration urines were lower in females than in males and, therefore, the gender differences were reduced after correcting concentrations by SG. A study involving a larger number of male and females subjects should be conducted in order to thoroughly study the effects of BUD administration on urine dilution, and to assess if these potential effects are genderdependent.

In conclusion, the assessment of the BUD metabolism after high-dose INH treatment demonstrates that false AAFs may be reported using the current WADA criterion. For this reason, the recommendation is to increase the reporting level to $40 \mathrm{ng} / \mathrm{mL}$ for $6 \beta O H B U D$ to detect BUD misuse in order to reduce the risk of reporting false AAFs. 


\section{Acknowledgements}

The authors are grateful for the financial support received from WADA (research grants 13D22RV) and Consell Català de l'Esport (Generalitat de Catalunya). Support from AGAUR (Generalitat de Catalunya) (2017 SGR 81) is also acknowledged.

\section{Conflict of interest}

The authors wish to disclose that there were no conflicts of interest associated with professional relationships, and that the results of the study are presented clearly, honestly, and without fabrication, falsification, or inappropriate data manipulation. 


\section{REFERENCES}

1. Brodgen RN, McTavish D. Budesonide. An updated review of its pharmacological properties, and therapeutic efficacy in asthma and rhinitis. Drugs 1992;44(3):375-407.

2. McKeage K, Goa KL. Budesonide (Entocort EC Capsules): a review of its therapeutic use in the management of active Crohn's disease in adults. Drugs 2002;62(15):2263-2282.

3. Duclos M. Evidence on ergogenic action of glucocorticoids as a doping agent risk. Physician Sportsmed 2010;38(3):121-7.

4. Keller-Wood ME, Dallman MF. Corticosteroid inhibition of ACTH secretion. Endocr $\operatorname{Rev} 1984 ; 5(1): 1-24$.

5. Henzen C, Suter A, Lerch E, et al. Suppression and recovery of adrenal response after short-term, high-dose glucocorticoid treatment. The Lancet 2000;355:542-45.

6. WADA Technical Document - TD2018MRPL [World Anti-Doping Agency Website]. Available at: https://www.wadaama.org/sites/default/files/resources/files/td2018mrpl_v1_finaleng.pdf (Accessed April 2019).

7. Matabosch X, Pozo OJ, Pérez-Mañá C, et al. Identification of budesonide metabolites in human urine after oral administration. Anal Bioanal Chem 2012;404(2):325-340.

8. Matabosch X, Pozo OJ, Pérez-Mañá C, et al. Discrimination of prohibited oral use from authorized inhaled treatment of budesonide in sports. Ther Drug Monit 2013;35(1):118-28.

9. WADA. 2012 Anti-Doping Testing Figures Report [World Anti-Doping Agency $\begin{array}{lll}\text { Website]. } & \text { Available } & \text { at: }\end{array}$ ama.org/sites/default/files/resources/files/WADA-2012-Anti-Doping-Testing-FiguresReport-EN.pdf (Accessed April 2019). 
10. WADA. 2017 Anti-Doping Testing Figures Report [World Anti-Doping Agency Website]. Available at: https://www.wadaama.org/sites/default/files/resources/files/2017_anti-doping_testing_figures_en_0.pdf (Accessed April 2019).

11. WADA Technical Document - TD2018DL [World Anti-Doping Agency Website]. Available at: https://www.wadaama.org/sites/default/files/resources/files/td2018dl_v1_en.pdf (Accessed April 2019). 12. Levine L, Fahy JP. Evaluation of urinary lead determinations. J Ind Hyg Toxicol 1996;20:379-391. 


\section{Tables}

Table 1. Clinical studies performed (see text for explanation).

\begin{tabular}{lllllll}
\hline study & type of study & $\mathrm{n}$ & $\mathrm{sex}$ & route & daily dose & urine collection $^{\mathrm{a}}$ \\
\hline 1 & cross-over & $4 / 4$ & $\mathrm{M} / \mathrm{F}$ & $\mathrm{INT}$ & $256 \mu \mathrm{g} \times 3$ days & 24-hour total urine up to day 4 \\
& & $4 / 4$ & $\mathrm{M} / \mathrm{F}$ & $\mathrm{INH}$ & $800 \mu \mathrm{g} \times 3$ days & 24-hour total urine up to day 4 \\
& & 4 & $\mathrm{M}$ & $\mathrm{INH}$ & $1600 \mu \mathrm{g} \times$ 3 days & 24-hour total urine up to day 4 \\
\cline { 2 - 7 } 2 & single-arm & 8 & $\mathrm{~F}$ & oral & $3 \mathrm{mg}$ & 24-hour total urine up to day 4 \\
\hline
\end{tabular}

a) Collection periods after administration are detailed in the text

Table 2. Subjects' characteristics.

\begin{tabular}{lllllll}
\hline study & sex & $\mathrm{n}$ & age (years) & height $(\mathrm{cm})$ & weight $(\mathrm{kg})$ & BMI $\left(\mathrm{kg} / \mathrm{m}^{2}\right)$ \\
\hline 1 & $\mathrm{~F}$ & 4 & $33 \pm 7$ & $170 \pm 2$ & $62 \pm 5$ & $22 \pm 2$ \\
& $\mathrm{M}$ & 4 & $22 \pm 2$ & $181 \pm 4$ & $76 \pm 10$ & $23 \pm 2$ \\
\cline { 2 - 6 } 2 & $\mathrm{~F}$ & 8 & $34 \pm 10$ & $164 \pm 5$ & $61 \pm 13$ & $23 \pm 5$ \\
\hline
\end{tabular}

Values are mean \pm SD.

Table 3. Analysis conditions of $6 \beta$ OHBUD, BUD and 16 $\alpha$ OHPRED by LC-MS/MS.

Retention time (RT), monoisotopic mass (MM), precursor and product ions (m/z), and collision energy (CE). The first product ion indicated for each metabolite was used for quantification purposes. Cone voltage was set to $20 \mathrm{~V}$ for all transitions.

\begin{tabular}{|c|c|c|c|c|c|}
\hline Metabolite & RT (min) & MM (Da) & Precursor ion & Product ion(s) & CE $(\mathrm{eV})$ \\
\hline 16 $\alpha$ OHPRED & 3.30 & 376 & 377 & $\begin{array}{l}226 \\
147 \\
159\end{array}$ & $\begin{array}{l}26 \\
30 \\
28\end{array}$ \\
\hline $6 \beta O H B U D$ & 4.65 & 446 & 447 & $\begin{array}{l}339 \\
121 \\
171\end{array}$ & $\begin{array}{l}14 \\
35 \\
35\end{array}$ \\
\hline BUD & 6.35 & 430 & 431 & $\begin{array}{l}147 \\
173 \\
323\end{array}$ & $\begin{array}{l}24 \\
26 \\
12\end{array}$ \\
\hline 16 $\alpha$ OHPRED-d5 & 3.20 & 381 & 382 & 229 & 26 \\
\hline 6ßOHBUD-d8 & 4.55 & 454 & 455 & 339 & 14 \\
\hline BUD-d8 & 6.25 & 438 & 439 & 173 & 20 \\
\hline
\end{tabular}


Table 4. Percentage of AAFs detected after intranasal treatment $(256 \mu \mathrm{g} / \mathrm{day} / 3$ days, INT), inhalation (400 $\mu \mathrm{g} /$ day/3days, INH 400; $800 \mu \mathrm{g} /$ day/3days, INH 800; 1600 $\mu \mathrm{g} /$ day/3days, INH 1600) and oral administration (single dose of $3 \mathrm{mg}$ ). Subjects' sex (males, M; females, F) and time (hours, h; days, d) are indicated. In A, reporting levels are established based on non-corrected concentrations. In B, reporting levels are established based on concentrations normalized by SG.

Results of INH 400 and oral administration in males were obtained in a previous study (8).

\begin{tabular}{|c|c|c|c|c|c|c|c|c|c|c|c|c|}
\hline & \multirow{3}{*}{ Compound } & & \multicolumn{10}{|c|}{ AAFs (\%) detected after administration of BUD by different routes } \\
\hline & & \multirow{2}{*}{$\begin{array}{c}\text { Reporting } \\
\text { level } \\
\text { (ng/mL) }\end{array}$} & \multicolumn{2}{|c|}{$\begin{array}{l}\text { INT } \\
(1-3 d)\end{array}$} & \multirow{2}{*}{$\begin{array}{c}\text { INH } 400 \\
(1-3 \mathrm{~d}) \\
\mathrm{M}^{\mathrm{a}}\end{array}$} & \multicolumn{2}{|c|}{$\begin{array}{c}\text { INH } 800 \\
(1-3 d)\end{array}$} & \multirow{2}{*}{$\begin{array}{l}\text { INH } 1600 \\
(1-3 d)\end{array}$} & \multicolumn{2}{|c|}{$\begin{array}{c}\text { oral } \\
(0-8 \mathrm{~h})\end{array}$} & \multicolumn{2}{|c|}{$\begin{array}{c}\text { oral } \\
(8-24 \mathrm{~h})\end{array}$} \\
\hline & & & $\mathrm{F}$ & $\mathrm{M}$ & & $\mathrm{F}$ & M & & $\mathrm{F}$ & $\mathrm{M}^{\mathrm{a}}$ & $\mathrm{F}$ & $\mathrm{M}^{\mathrm{a}}$ \\
\hline \multirow[t]{5}{*}{$\mathrm{A}$} & $6 \beta \mathrm{OHBUD}$ & 30 & 0 & 0 & 0 & 0 & 3 & 3 & 44 & 92 & 25 & 50 \\
\hline & & 40 & 0 & 0 & 0 & 0 & 0 & 0 & 38 & 69 & 19 & 44 \\
\hline & BUD & 30 & 0 & 0 & 0 & 0 & 3 & 3 & 25 & 62 & 0 & 21 \\
\hline & & 50 & 0 & 0 & 0 & 0 & 0 & 0 & 6 & 31 & 0 & 6 \\
\hline & 16 $\alpha$ OHPRED & 450 & 0 & 0 & 0 & 0 & 0 & 0 & 13 & 31 & 0 & 6 \\
\hline \multirow[t]{4}{*}{ B } & $6 \beta \mathrm{OHBUD}$ & 30 & 0 & 0 & 0 & 0 & 8 & 6 & 81 & 81 & 31 & 69 \\
\hline & & 50 & 0 & 0 & 0 & 0 & 0 & 0 & 44 & 44 & 19 & 31 \\
\hline & BUD & 50 & 0 & 0 & 0 & 0 & 0 & 0 & 31 & 25 & 0 & 6 \\
\hline & 16 $\alpha$ OHPRED & 450 & 0 & 0 & 0 & 0 & 0 & 0 & 56 & 31 & 0 & 6 \\
\hline
\end{tabular}




\section{Figure captions}

Figure 1. Concentrations of $6 \beta$ OHBUD, BUD, and 16 $\alpha$ OHPRED after intranasal treatment (256 $\mu \mathrm{g} /$ day/3days, INT) and inhalation (800 $\mu \mathrm{g} /$ day/3days, INH 800; 1600 $\mu \mathrm{g} /$ day/3days, INH 1600). Time (days, d) and subjects' sex (female, F; male, M) are noted. Dotted line corresponds to the reporting level of $30 \mathrm{ng} / \mathrm{mL}$ for GCs.

Figure 2. Urinary excretion profiles (ng/min, mean $+\mathrm{SD}$ ) of $6 \beta \mathrm{OHBUD}, \mathrm{BUD}$ and 16aOHPRED in samples collected after intranasal (INT), inhaled (INH) and oral administrations. Dotted line corresponds to females and full line corresponds to males. Arrows indicate administrations. Data included involve results from a previous study where BUD was orally administered to males (8).

Figure 3. Concentrations of $6 \beta$ OHBUD, BUD and 16 $\alpha$ OHPRED after oral administration. Time (hours, h) and subjects' sex (female, F; male, M) are noted. Dotted line corresponds to the reporting level of $30 \mathrm{ng} / \mathrm{mL}$ for GCs. Data included involve results from a previous study where BUD was orally administered to males (8). 